\title{
Advantages of externally powered prosthesis with feedback system using pseudo-cineplasty
}

\author{
Seiji Nambu, MD; ${ }^{1}$ Mitsuhiko Ikebuchi, MD; ${ }^{2}$ Masashi Taniguchi; ${ }^{3}$ Choong Sik Park; ${ }^{3}$ Takahiro Kitagawa; $^{3}$ \\ Shigeyoshi Nakajima, PhD; ${ }^{4}$ Tatsuya Koike, $\mathbf{M D}, \mathbf{P h D}^{5^{*}}$ \\ Departments of ${ }^{1}$ Orthopaedic Surgery and ${ }^{2}$ Rehabilitation, Osaka City University Medical School, Osaka, Japan; \\ ${ }^{3}$ Control Engineering and Electronic Materials, Technology Research Institute of Osaka Prefecture, Izumi, Japan; \\ ${ }^{4}$ Department of Information and Communication Engineering, Osaka City University Graduate School of Engineering, \\ Osaka, Japan; ${ }^{5}$ Department of Rheumatosurgery, Osaka City University Medical School, Osaka, Japan
}

\begin{abstract}
Externally powered upper-limb prostheses are difficult to use because of the lack of sensory feedback. Neuroprostheses have recently been developed for people with upper-limb amputation but are complicated, expensive, and still developing. We therefore designed a simple system by combining pseudo-cineplasty with extended physiological proprioception to provide sensory feedback to the body. We penetrated the palmaris longus tendon percutaneously with a metal ring, similar to that used in body piercing, in a nondisabled subject as a pseudo-cineplasty. The tendon and ring were connected to the system, and a sensory feedback experiment was performed. We investigated the ability of the user to determine the size of an object grasped by the prosthetic hand without visual information. The subject could distinguish between large and small objects with 100\% accuracy and between small, medium, and large objects with $80 \%$ accuracy. In pseudo-cineplasty, control and sensory feedback are natural because the prosthetic hand is controlled by muscle contraction. Tension transmitted from the prosthetic hand is sensed via muscle spindles and skin sensors. This technique allows only partial sensory feedback but appears to offer several advantages over other human-machine interfaces.
\end{abstract}

Key words: amputation, cineplasty, extended physiological proprioception, externally powered prosthesis, human-machine interface, muscle spindles, myoelectric prosthesis, sensory feedback, transinformation, upper limb.

\section{INTRODUCTION}

The most commonly used externally powered upperlimb prostheses are myoelectric hand prostheses. Users of many of these devices experience erroneous movements at some times and generally cannot perform movements as desired without considerable practice [1]. Moreover, the absence of sensory feedback means that the user must visually confirm whether the hand component of the prosthesis is open, closed, or grasping an object. Such prosthetic hands are thus difficult to use [1], but more physiological externally powered upper-limb prostheses are still in the developmental stage [2]. Various research institutions have been investigating methods for moving myoelectric hand prostheses in ways that are not as susceptible to erroneous activation and can provide sensory feedback [3-6]. For example, vibration or electric stimulation applied to the amputated arm while grasping an object with the prosthetic hand can provide

\footnotetext{
Abbreviations: EPP = extended physiological proprioception. *Address all correspondence to Tatsuya Koike, MD, PhD; Center for Senile Degenerative Disorders, Osaka City University Medical School, 1-4-3 Asahi-machi, Abeno-ku, Osaka 5454-8585, Japan; +81-6-6646-6010; fax: +81-6-6646-6010. Email: tatsuya@med.osaka-cu.ac.jp http://dx.doi.org/10.1682/JRRD.2013.03.0067
} 
the user with information [7-9]. However, combining a sensory feedback structure with the already extremely complex system of a myoelectric prosthetic hand further increases the complexity of the overall structure. The ideal situation would be an externally powered prosthetic hand with a simple structure that can be controlled without erroneous activation and provides sensory feedback.

Muscles and tendons can be connected to external interfaces by employing the existing surgical technique of cineplasty [10-12]. This surgical technique was previously employed to operate mechanical prosthetic hands by directly attaching a cable to a surgically modified muscle in the amputated upper limb.

Movement of the hand component is linked to muscle via the interface and to associated muscle spindles and skin sensors. In muscle tunnel cineplasty [13] or tendon external cineplasty [14], muscle and tendon forces can be transmitted externally from the body. The concept of combining control and feedback through a direct link between physiological movement and movement of an externally powered prosthesis was pioneered by Simpson [15] under the name of extended physiological proprioception (EPP) and has been previously reported for electric-powered prostheses [16-18]. Regarding the use of a combination of cineplasty and an externally powered prosthesis as a control method, Weir et al. states, "We believe that direct connections that are simple, effective, and comfortable are possible if surgeons, rehabilitation doctors, engineers, prosthetists, etc., work together collaboratively on the control problem" [19].

Weir et al. performed an experiment using cineplasty to control externally powered prostheses and achieved "good control of the prosthesis" [19]. Although the system devised and tested by Weir et al. incorporated sensory feedback, they did not provide experimental evidence of the use of that feedback other than in control of the action of the prosthesis [19]. Furthermore, they used relatively large muscles to control the externally powered prostheses: a flexor tendon for closing and an extensor tendon for opening the prosthetic hand. We therefore performed an experiment to determine whether users could distinguish between large and small objects grasped by the prosthetic hand without visual confirmation via pseudo-cineplasty using a tendon from a single small, weak muscle. Weir et al. showed that control of an electric-powered prosthetic hand was feasible [19], and refining that system for practical application might be relatively easy.

\section{METHODS}

The first step was to prepare a system to achieve EPP control. Positional information on muscle movement is linked, in a one-to-one relationship, with the opening and closing of the electromechanical hand. Information on the level of opening or closing of the hand is transmitted to the muscle via the interface (Figure 1). We then prepared an interface that detects the distance of muscle contraction or relaxation and an electromechanical hand that receives this information and provides movement (opening or closing) in proportion to the magnitude of contraction or relaxation. In terms of the electromechanical hand, we initially attempted to modify the hand component of a commercially available myoelectric prosthetic hand (OttoBock; Duderstadt, Germany). However, adjusting the opening and closing levels to the requisite distances and sending this information to the interface proved difficult. We therefore prepared an entirely original electromechanical hand (Figure 2) that, due to budgetary constraints and construction limitations, was larger than desirable. We mapped excursion of the palmaris longus tendon to the opening-and-closing angle of the electromechanical hand: full flexion of the wrist to achieve full closure of the mechanical hand and full extension of the wrist to achieve full opening of the mechanical hand. The gripper can be opened to a maximum of $10 \mathrm{~cm}$. The time required to go from fully open to fully closed is $0.4 \mathrm{~s}$. Maximum grip force was designed to be $25 \mathrm{~N}$.

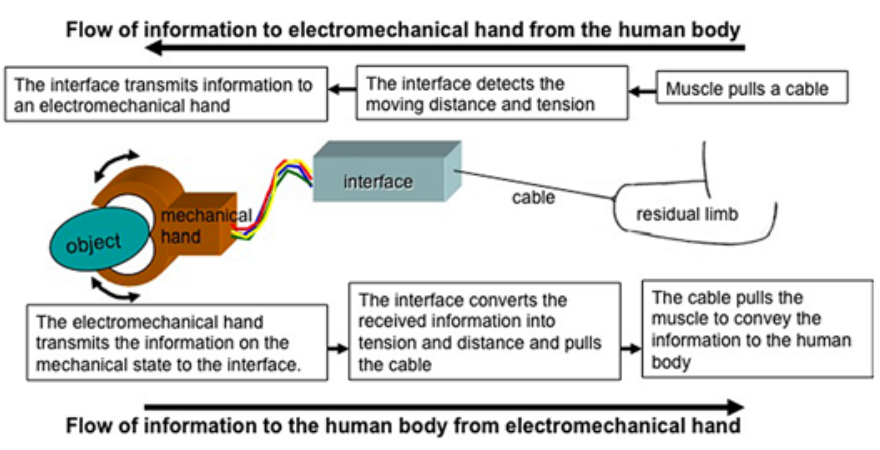

Figure 1.

Flow of information between electrical hand and human body. Intention of user is transmitted to prosthetic hand via interface as muscle pulls cable. Information on state of prosthetic hand is transmitted to residual upper limb via interface. 


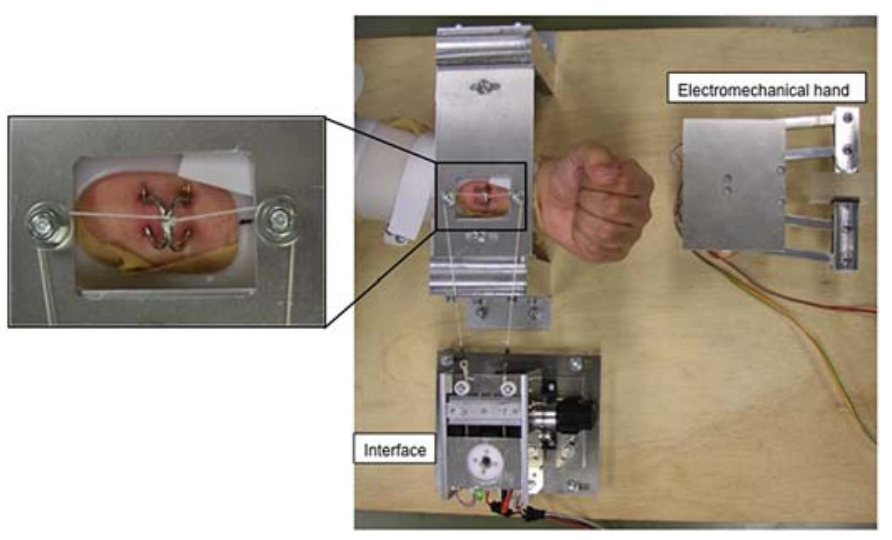

Figure 2.

Overview of system. Ring is connected directly to palmaris longus tendon. Thread connects ring to interface. Interface and electromechanical hand are connected via personal computer.

Although the system should be connected to the human body, no patients with upper-limb amputation who had undergone cineplasty were available. A hole was therefore made percutaneously in the left palmaris longus tendon of one of the authors (S.N.). A metal ring was passed through this hole in order to prepare the pseudo-cineplasty (Figure 2).

The prepared interface was connected to this pseudocineplasty (Figure 2). The electromechanical hand was set to close and open when the palmaris longus shortened and lengthened, respectively (Figure 3).

Confirmation was made that the electromechanical hand would close and open when the subject shortened and lengthened the palmaris longus. The prosthetic hand was then hidden from the subject's view and made to hold large and small (widths of 8 and $2 \mathrm{~cm}$, respectively) or large, medium, and small (widths of 8,5 , and $2 \mathrm{~cm}$, respectively) objects to investigate whether the size of the object grasped could be determined in the absence of visual information (Figure 4). This size-determination trial was repeated 20 times for each group of objects. Beforehand, the subject performed 10 trials without visual feedback to check the operation of the system. Presentation of objects during the size-determination trials was performed at random by the examiner.

\section{RESULTS}

Excursion of the tendon according to motion of the thread was $12 \mathrm{~mm}$. Maximum force as detected by sensor
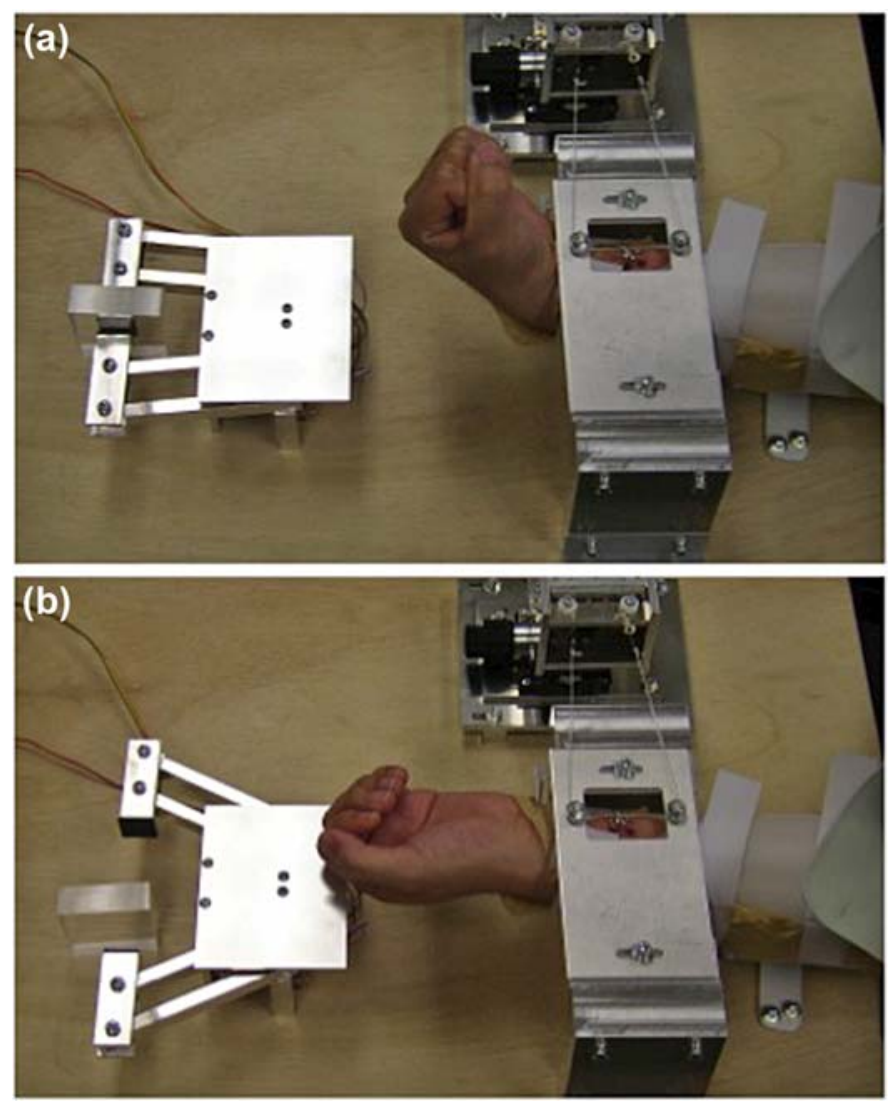

Figure 3.

Movement of electromechanical hand. Electromechanical hand (a) closes and (b) opens when palmaris longus shortens and lengthens, respectively.

was $1.6 \mathrm{~N}$ (approximately $160 \mathrm{~g}$ ). Due to the looseness of the piercing portion, these values might be approximate. Large and small objects were able to be distinguished in 100 percent of trials (Table 1), while large, medium, and small objects were able to be distinguished in 80 percent of trials (Table 2). As shown in Table 2, errors were made only in the first half of the trials. Accuracy rose to 100 percent in the latter half of the experiment. Information on the change in the length of the palmaris longus was not accurately transmitted to the interface because the piercing-like ring inclined in the shortening and lengthening direction of the muscle and the thread connecting the ring was made of a slightly elastic material. This led to marked errors in the perception of the force produced. Despite this, control was possible and differences in the sizes of grasped objects could be judged in the absence of visual information. The hardness of an object could not be determined due to the 
JRRD, Volume 51, Number 7, 2014

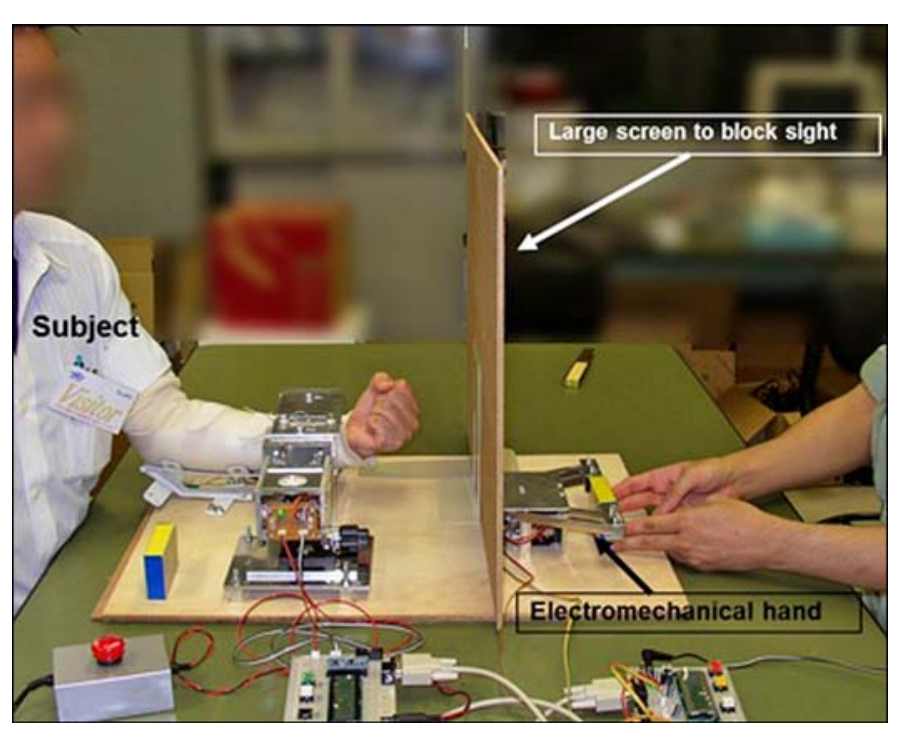

Figure 4.

Determining size of object grasped in absence of visual confirmation. Large plate was positioned between subject and electromechanical hand so that subject could not see movements of hand. Ability of subject to determine size of object grasped, without visual input, was investigated.

poor connection between the muscle and interface and the low precision of the interface.

\section{DISCUSSION}

When deep sensation is lost in the limbs due to peripheral nerve injury or cerebral disease, symptoms of ataxia develop and limb functions decrease markedly despite the maintenance of muscle strength. When sensory information from the hand does not reach the cranial nerves due to the loss of deep sensation, the individual cannot feel the hand opening and clenching and visual confirmation is required to ensure that an object is being grasped. The condition of a person with upper-limb amputation using a myoelectric prosthetic hand can be considered analogous to this loss of deep sensation. While methods are available to control the opening and closing of a prosthetic hand, systems are not yet available to determine the state of the hand other than through visual confirmation. Moreover, a myoelectric prosthetic hand is not part of the body and may move erroneously at times, making operation more difficult than using a neurologically ataxic hand. Clues
Table 1.

Results for distinguishing two sizes (large and small) of objects. Large and small objects were successfully distinguished in all trials.

\begin{tabular}{rcc}
\hline Trial No. & Size of Object & Subject Answer \\
\hline 1 & Small & Small \\
2 & Small & Small \\
3 & Small & Small \\
4 & Large & Large \\
5 & Large & Large \\
6 & Small & Small \\
7 & Large & Large \\
8 & Large & Large \\
9 & Small & Small \\
10 & Large & Large \\
11 & Small & Small \\
12 & Small & Small \\
13 & Large & Large \\
14 & Small & Small \\
15 & Large & Large \\
16 & Large & Large \\
17 & Large & Large \\
18 & Small & Small \\
19 & Large & Large \\
20 & Small & Small \\
\hline \hline
\end{tabular}

Table 2.

Results for distinguishing three sizes (large, medium, and small) of objects. Italics represent incorrect answers. Overall accuracy was 80 percent. Incorrect answers were observed only in early trials, with correct answers in all later trials.

\begin{tabular}{lll}
\hline Trial No. & Size of Object & Subject Answer \\
\hline 1 & Small & Medium \\
2 & Medium & Medium \\
3 & Large & Medium \\
4 & Medium & Small \\
5 & Small & Small \\
6 & Large & Medium \\
7 & Medium & Medium \\
8 & Large & Large \\
9 & Small & Small \\
10 & Medium & Medium \\
11 & Small & Small \\
12 & Large & Large \\
13 & Medium & Medium \\
14 & Medium & Medium \\
15 & Large & Large \\
16 & Medium & Medium \\
17 & Small & Small \\
18 & Small & Small \\
19 & Medium & Medium \\
20 & Large & Large \\
\hline \hline
\end{tabular}


toward solving this problem can be found in the cineplasties carried out in the past.

Our procedure was not strictly a cineplasty. We therefore call our piercing method "pseudo-cineplasty." Our system was operable using the small amount of force generated by the palmaris longus muscle, suggesting that other such small muscles could achieve similar results. The low level of force obtained by directly connecting a small muscle to the system by pseudo-cineplasty may be sufficient to operate a prosthetic hand with appropriate sensors. The maximum contractile force detectable in this experiment was only about $1.6 \mathrm{~N}$. Muscles capable of exerting $160 \mathrm{~g}$ can thus be considered sufficient to operate this system.

The procedure itself was very simple: the tendon and skin were collectively penetrated using a piercing then connected to the interface. A similarly simple method may achieve results comparable with those of conventional cineplasty in people with upper-limb amputation. In addition, downsizing of the system may allow individual movements of multiple fingers of the hand. Sensory feedback from each finger may then allow the user to determine not only the size but also the shape of objects grasped by the prosthetic hand. The present system was larger than desired, but downsizing should prove relatively straightforward. The use of more precise devices in the system may enable determination of the hardness of a grasped object.

In cineplasty, the system is connected to muscle and tendon via a skin tunnel. Tactile and pressure sensations thus contribute to the perception of force and movement. The prosthetic hand designed in this study cannot provide a complete substitute for lost upper-limb function, but the size of grasped objects might be perceived without visual compensation, unlike the situation with current prosthetic hands. Contractile force and length of the palmaris longus could not be accurately determined using the interface, because the connection between the human body and the system was very loose and the detected muscle force was far lower than the actual value [18].

Use of this type of prosthetic hand requires surgery, and to allow individual movement of fingers, multiple pseudocineplasty procedures would need to be performed to access the flexor and extensor tendons. Although the piercing applied in the present study might be considered minimally invasive, clarification is needed regarding whether a sufficient number of exteriorizations could be applied to the amputated limb. Piercing a tendon percutaneously would also carry a relatively high long-term risk of infection. Other methods for creating suitable connections between tendon and interface therefore need to be developed, such as magnetic connections via skin. Although various promising human-machine interfaces are currently being investigated [2,20-25], an EPP system with a cineplastycontrolled electromechanical prosthetic hand may be a more practical option to provide sensory feedback at present. Such a system would control the prosthetic hand via muscle contraction and would sense the tension and length changes transmitted from the prosthetic hand via muscle spindles and skin sensors, allowing more natural sensory feedback and control of the prosthetic device.

Some limitations need to be considered when interpreting the present results. Whether similar results could be obtained for an individual with an amputation remains unclear because the palmaris longus of a subject with an intact hand was used in this investigation. Individual differences in the ability to feel differences in size presumably exist. Furthermore, performing this experiment in people with amputation may prove difficult, because cineplasty is no longer performed. However, according to the results of an earlier study by Weir et al. [19], similar results may be obtained if a muscle or tendon were exteriorized and connected to an EPP-type electromechanical prosthetic hand in people with upper-limb amputation.

This system combining cineplasty and EPP control may also be applicable for other limb prostheses. It may enable voluntary control of the prosthetic knee in patients with transfemoral amputation and control of the anklejoint prosthesis in patients with transtibial amputation. The EPP control provided through the cineplasty connection could produce more natural responses in an electricpowered prosthetic knee or ankle. The more natural response would facilitate walking on uneven ground and climbing or descending stairs. The application of cineplasty to a movable muscle in patients with cervical spinal cord injury and palsy of all four limbs may also be useful to control various devices. In addition to usefulness in hand and leg prostheses, an interface employing cineplasty may also provide utility in human-machine interfaces in the future.

\section{CONCLUSIONS}

In our single-subject experiment, we proved the ability of the subject to determine the size of an object grasped by 
the prosthetic hand without visual information via pseudocineplasty using a single tendon from a small, weak muscle. Piercing the palmaris longus tendon through the skin as a pseudo-cineplasty appears to be a novel method. Our idea is derived from the original cineplasties carried out in the past and has several weak points, such as infection. However, we believe a more physiological humanmachine interface could be built by developing our idea.

\section{ACKNOWLEDGMENTS}

\author{
Author Contributions: \\ Study concept and design: S. Nambu, M. Ikebuchi, T. Koike. \\ Acquisition of data: S. Nambu, M. Ikebuchi, M. Taniguchi, C. S. Park, \\ T. Kitagawa, S. Nakajima. \\ Analysis and interpretation of data: S. Nambu, M. Ikebuchi, T. Koike. \\ Direct oversight of data collection and analysis: S. Nambu, T. Koike. \\ Drafting of manuscript: S. Nambu, T. Koike. \\ Critical revision of manuscript for important intellectual content: \\ T. Koike. \\ Principal investigator: S. Nambu. \\ Obtained funding: T. Koike.
}

Financial Disclosures: The authors have declared that no competing interests exist.

Funding/Support: This material was unfunded at the time of manuscript preparation.

Additional Contributions: We would like to thank the numerous individuals whose enormous support and insightful comments proved invaluable during the course of this study. Finally, we gratefully appreciate the support of the Technology Research Institute of Osaka Prefecture, which enabled completion of our thesis. Dr. Nambu is now with the Department of Rehabilitation, Okuma Central Hospital, Osaka, Japan. Dr. Koike is now with the Center for Senile Degenerative Disorders, Osaka City University Medical School, Osaka, Japan, and the Search Institute for Bone and Arthritis Disease, Shirahama Foundation for Health and Welfare, Wakayama, Japan.

Institutional Review: Because the subject of this study was one of the authors, we judged that no institutional review was required.

\section{REFERENCES}

1. Dudkiewicz I, Gabrielov R, Seiv-Ner I, Zelig G, Heim M. Evaluation of prosthetic usage in upper limb amputees. Disabil Rehabil. 2004;26(1):60-63. [PMID:14660200] http://dx.doi.org/10.1080/09638280410001645094

2. Tabot GA, Dammann JF, Berg JA, Tenore FV, Boback JL, Vogelstein RJ, Bensmaia SJ. Restoring the sense of touch with a prosthetic hand through a brain interface. Proc Natl Acad Sci USA. 2013;110(45):18279-84. Erratum in: Proc Natl Acad Sci USA. 2014;111(2):875. [PMID:24127595] http://dx.doi.org/10.1073/pnas.1221113110
3. Antfolk C, Balkenius C, Lundborg G, Rosén B, Sebelius F. Design and technical construction of a tactile display for sensory feedback in a hand prosthesis system. Biomed Eng Online. 2010;9:50. [PMID:20840758] http://dx.doi.org/10.1186/1475-925X-9-50

4. Rosén B, Ehrsson HH, Antfolk C, Cipriani C, Sebelius F, Lundborg G. Referral of sensation to an advanced humanoid robotic hand prosthesis. Scand J Plast Reconstr Surg Hand Surg. 2009;43(5):260-66. [PMID:19863429] http://dx.doi.org/10.3109/02844310903113107

5. Pylatiuk C, Kargov A, Schulz S. Design and evaluation of a low-cost force feedback system for myoelectric prosthetic hands. J Prosthet Orthot. 2006;18:57-61. http://dx.doi.org/10.1097/00008526-200604000-00007

6. Kyberd PJ, Mustapha N, Carnegie F, Chappell PH. A clinical experience with a hierarchically controlled myoelectric hand prosthesis with vibro-tactile feedback. Prosthet Orthot Int. 1993;17(1):56-64. [PMID:8337101] http://dx.doi.org/10.3109/03093649309164355

7. Okuno R, Yoshida M, Uchiyama T, Akazawa K. [Basic study of vibrotactile sensory feedback system for biomimetic powered-prosthetic hand]. IEEJ Trans EIS. 1996; 116:1246-51. Japanese.

8. Okuno R, Yoshida M, Uchiyama T, Akazawa K. [Infrasonic vibrotactile sensory feedback system for the myoelectric hand prosthesis]. IEICE technical report. MBE. 1997;96: 99-106. Japanese.

9. Yada K, Saihara M, Yoshida M. [A study of sensory feedback system for two kinds of information transmission of EMG prosthetic hand]. IEICE technical report. MBE. 2004; 104:11-14. Japanese.

10. Spittler AW, Rosen IE. Cineplastic muscle motors for prostheses of arm amputees. J Bone Joint Surg Am. 1951;33-A(3): 601-11, passim. [PMID:14850498]

11. Mazet R Jr. Cineplasty; historical review, present status, and critical evaluation of sixty-four patients. J Bone Joint Surg Am. 1958;40-A(6):1389-1400. [PMID:13610968]

12. Brav EA, Fletcher MJ, Kuitert JH, Leonard F, Luscombe HB, MacDonald WF, Spittler AW, Vultee FE Jr, Woodard GH. Cineplasty; an end-result study. J Bone Joint Surg Am. 1957;39-A(1):59-76. [PMID:13385264]

13. Brückner L. Sauerbruch-Lebsche-Vanghetti cineplasty: The surgical procedure. Orthop Traumatol. 1992;1:90-99. http://dx.doi.org/10.1007/BF02620403

14. Beasley RW. The tendon exteriorization cineplasty, a preliminary report. Inter-clin Inf Bull. 1966;5:6-8.

15. Simpson DC. The choice of control system for the multimovement prosthesis: extended physiological proprioception (EPP). In: Herberts P, editor. The control of upper-extremity prostheses and orthoses. Springfield (IL): Charles Thomas; 1974. 
16. Doubler JA, Childress DS. An analysis of extended physiological proprioception as a prosthesis-control technique. J Rehabil Res Dev. 1984;21(1):5-18. [PMID:6527290]

17. Doubler JA, Childress DS. Design and evaluation of a prosthesis control system based on the concept of extended physiological proprioception. J Rehabil Res Dev. 1984; 21(1):19-31. [PMID:6527287]

18. Farrell TR, Weir RF, Heckathorne CW, Childress DS. The effects of static friction and backlash on extended physiological proprioception control of a powered prosthesis. J Rehabil Res Dev. 2005;42(3):327-41. [PMID:16187245] http://dx.doi.org/10.1682/JRRD.2004.05.0052

19. Weir RF, Heckathorne CW, Childress DS. Cineplasty as a control input for externally powered prosthetic components. J Rehabil Res Dev. 2001;38(4):357-63. [PMID:11563487]

20. Leuthardt EC, Schalk G, Moran D, Ojemann JG. The emerging world of motor neuroprosthetics: A neurosurgical perspective. Neurosurgery. 2006;59(1):1-14, discussion 1-14. [PMID:16823294] http://dx.doi.org/10.1227/01.NEU.0000221506.06947.AC

21. Zhou P, Lowery MM, Englehart KB, Huang H, Li G, Hargrove L, Dewald JP, Kuiken TA. Decoding a new neural machine interface for control of artificial limbs. J Neurophysiol. 2007;98(5):2974-82. [PMID:17728391] http://dx.doi.org/10.1152/jn.00178.2007

22. McKhann GM 2nd. Cortical control of a prosthetic arm for self-feeding. Neurosurgery. 2008;63(2):N8-9. [PMID:18797344] http://dx.doi.org/10.1227/01.NEU.0000335797.80384.06

23. Gupta R, Ashe J. Offline decoding of end-point forces using neural ensembles: Application to a brain-machine interface. IEEE Trans Neural Syst Rehabil Eng. 2009;17(3):254-62.
[PMID:19497832]

http://dx.doi.org/10.1109/TNSRE.2009.2023290

24. Matsuoka Y, Afshar P, Oh M. On the design of robotic hands for brain-machine interface. Neurosurg Focus. 2006; 20(5):1-9. [PMID:16711660]

http://dx.doi.org/10.3171/foc.2006.20.5.4

25. Hatsopoulos NG, Donoghue JP. The science of neural interface systems. Annu Rev Neurosci. 2009;32:249-66.

[PMID:19400719]

http://dx.doi.org/10.1146/annurev.neuro.051508.135241

Submitted for publication August 13, 2013. Accepted in revised form March 18, 2014.

This article and any supplementary material should be cited as follows:

Nambu S, Ikebuchi M, Taniguchi M, Park CS, Kitagawa T, Nakajima S, Koike T. Advantages of externally powered prosthesis with feedback system using pseudo-cineplasty. J Rehabil Res Dev. 2014;51(7):1095-1102.

http://dx.doi.org/10.1682/JRRD.2013.03.0067

ResearchID/ORCID: Seiji Nambu, MD: L-3947-2014; Mitsuhiko Ikebuchi, MD: L-4808-2014; Shigeyoshi Nakajima, PhD: L-4400-2014; Tatsuya Koike, MD, PhD: L5533-2014

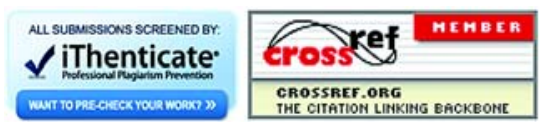


